Bryan D. James, PhD

Sue E. Leurgans, $\mathrm{PhD}$

Liesi E. Hebert, ScD

Paul A. Scherr, PhD, ScD

Kristine Yaffe, MD

David A. Bennett, MD

Correspondence to

Dr. James:

Bryan_James@rush.edu

\title{
Contribution of $\mathrm{Alzheimer}$ disease to mortality in the United States
}

\section{ABSTRACT}

Objective: To assess the burden of mortality attributable to Alzheimer disease (AD) dementia in the United States.

Methods: Data came from 2,566 persons aged 65 years and older (mean 78.1 years) without dementia at baseline from 2 cohort studies of aging with identical annual diagnostic assessments of dementia. Because both studies require organ donation, ascertainment of mortality was complete and dates of death accurate. Mortality hazard ratios (HRs) after incident AD dementia were estimated per 10-year age strata from proportional hazards models. Population attributable risk percentage was derived to estimate excess mortality after a diagnosis of $A D$ dementia. The number of excess deaths attributable to $A D$ dementia in the United States was then estimated.

Results: Over an average of 8 years, 559 participants (21.8\%) without dementia at baseline developed AD dementia and 1,090 (42.4\%) died. Median time from AD dementia diagnosis to death was 3.8 years. The mortality HR for AD dementia was 4.30 (confidence interval $=3.33,5.58$ ) for ages $75-84$ years and 2.77 (confidence interval $=2.37,3.23$ ) for ages 85 years and older (too few deaths after $A D$ dementia in ages $65-74$ were available to estimate $H R$ ). Population attributable risk percentage was $37.0 \%$ for ages $75-84$ and $35.8 \%$ for ages 85 and older. An estimated 503,400 deaths in Americans aged 75 years and older were attributable to AD dementia in 2010.

Conclusions: A larger number of deaths are attributable to AD dementia in the United States each year than the number (<84,000 in 2010) reported on death certificates. Neurology ${ }^{\circledR} 2014 ; 82: 1045-1050$

\section{GLOSSARY}

$\mathbf{A D}=$ Alzheimer disease; $\mathbf{C D C}=$ Centers for Disease Control and Prevention; $\mathbf{H R}=$ hazard ratio; $\mathbf{P A R} \%=$ population attributable risk percentage.

Alzheimer disease $(\mathrm{AD})$ is listed by the Centers for Disease Control and Prevention (CDC) as the sixth leading cause of death in the United States, accounting for 83,494 deaths in 2010. ${ }^{1}$ This number is derived from death certificates, which are known to underreport persons dying of dementia. ${ }^{2}$ Up to 5 million Americans are currently living with $\mathrm{AD}$ dementia, ${ }^{3,4}$ a disease with an average time from diagnosis to death of 3 to 9 years. ${ }^{2,5}$ Given these figures, the burden of mortality attributable to $\mathrm{AD}$ dementia is potentially much higher than the numbers posted by the $\mathrm{CDC}$. A valid estimate of the number of deaths attributable to $\mathrm{AD}$ dementia would aid assessment of the societal burden of $\mathrm{AD}$ dementia, informing government and private research priorities and the development of the recently enacted National Alzheimer's Plan. ${ }^{6}$

Prospective follow-up of population-based cohorts can provide the most valid estimates of AD dementia incidence and is generally considered the best source for determining risk of mortality from $\mathrm{AD}$ dementia., ${ }^{2,47-10}$ Many people with $\mathrm{AD}$ dementia do not come to the attention of the health care system. ${ }^{11}$ Therefore, studies of medical records miss deaths from AD dementia. Furthermore, estimates based on observation of prevalent, rather than incident, cases may underestimate mortality risk by not including rapidly progressive $\mathrm{AD}$ dementia. ${ }^{12,13}$ The study objective was to estimate the risk of mortality attributable to incident $\mathrm{AD}$ dementia in 2 community-based

From the Rush Alzheimer's Disease Center (B.D.J., S.E.L., D.A.B.), Chicago; Departments of Internal Medicine (B.D.J.) and Neurological Sciences (S.E.L., D.A.B.), and Rush Institute on Healthy Aging (L.E.H., P.A.S.), Rush University Medical Center, Chicago, IL; Departments of Psychiatry, Neurology, and Epidemiology (K.Y.), University of California, San Francisco; and San Francisco Veterans Affairs Medical Center (K.Y.), San Francisco, CA. Go to Neurology.org for full disclosures. Funding information and disclosures deemed relevant by the authors, if any, are provided at the end of the article. 
cohort studies and produce an estimate of the number of deaths attributable to $\mathrm{AD}$ dementia in the United States.

METHODS Subjects. Two ongoing cohort studies of aging and $\mathrm{AD}$, the Religious Orders Study ${ }^{14}$ and the Rush Memory and Aging Project, ${ }^{15}$ provided the data for these analyses. In both studies, participants without known dementia at baseline agreed to annual detailed clinical evaluation and brain donation at the time of death. Participants in the Religious Orders Study are older Catholic nuns, priests, and brothers from across the United States. From January 1994 through February 2013, 1,168 persons aged 65 years and older were recruited into the study and completed a baseline evaluation. Participants in the Rush Memory and Aging Project are older, community-dwelling persons from retirement communities and subsidized senior housing facilities across Illinois. From September 1997 through February 2013, 1,574 persons completed a baseline evaluation. Clinical and diagnostic procedures are identical across the 2 studies, allowing them to be pooled for analysis. ${ }^{16}$ Follow-up among living participants exceeds $90 \%$. Although participants in these studies did not have known dementia at the time of recruitment, a small portion $(\mathrm{n}=176,6.4 \%)$ were diagnosed with dementia upon baseline clinical examination and were excluded from these analyses, leaving 2,566 for analysis. The baseline evaluation for both studies included self-reported date of birth (used to determine age), sex, race/ethnicity (reported here as non-Hispanic white vs other), and education (years of schooling completed). Compared with the general population of older people in the United States, our study sample had proportionately fewer males and persons of minority race, and participants were more highly educated.

Standard protocol approvals, registrations, and patient consents. Both studies were approved by the Institutional Review Board of the Rush University Medical Center, and informed consent was obtained from all participants.

Clinical evaluation and diagnosis of $\mathrm{AD}$ dementia. Annual clinical evaluations included medical history, neurologic examination, and cognitive testing. ${ }^{17,18}$ The medical history included questions regarding vascular disease history (e.g., claudication, stroke, and heart conditions) and vascular risk factors (hypertension, diabetes mellitus, and smoking); each were summed for analysis, range: $0-3$. Clinical diagnosis of $\mathrm{AD}$ and other dementias at each assessment was performed using a 3-stage process with computer scoring of cognitive tests followed by clinical judgment by a neuropsychologist, and diagnostic classification by an experienced clinician. ${ }^{17,18}$ Diagnosis of dementia and probable $\mathrm{AD}$ dementia followed National Institute of Neurological and Communicative Disorders and Stroke-Alzheimer's Disease and Related Disorders Association criteria. ${ }^{19}$ Eighty-eight percent of persons diagnosed with $\mathrm{AD}$ dementia who died in these cohorts were given a pathologic diagnosis of $\mathrm{AD}$ on postmortem examination using National Institute on Aging-Reagan criteria and blinded to clinical data. ${ }^{20}$

Ascertainment of mortality. Both cohort studies are autopsy studies requiring brain and tissue donation. The autopsy rate is nearly $90 \%$, thus for the majority of participants who die, the exact day of death is known. In addition to the annual clinical evaluations, participants are contacted regularly to determine vital status, and death is occasionally detected during quarterly contacts with an informant. Finally, the Social Security Death Index is regularly searched for the small number of participants we are unable to contact. Therefore, ascertainment of mortality is essentially complete, and dates of death are accurate.
Statistical analysis. We first compared persons who had an incident $\mathrm{AD}$ dementia diagnosis during follow-up with persons who did not for demographics and other covariates using $t$ tests or $\chi^{2}$ tests. Kaplan-Meier curves were obtained for 3 age strata (65-74 years, 75-84 years, and 85 years and older) to estimate the median time to death after a diagnosis of $\mathrm{AD}$ dementia. Mortality hazard ratios (HRs) for incident $\mathrm{AD}$ dementia were derived for the 3 age strata from proportional hazards models with age as the time scale, and participants entering the analysis at their baseline age (left truncation). ${ }^{21} \mathrm{AD}$ dementia status was treated as a time-varying absorbing state. Person-years for subjects who developed $\mathrm{AD}$ dementia contributed both to non- $\mathrm{AD}$ dementia (up to diagnosis) person-years and $\mathrm{AD}$ dementia (after diagnosis) person-years. Models included terms for sex, race, education, and parent study. We also present a model further adjusted for vascular comorbidities. Finally, we replaced the term for $\mathrm{AD}$ dementia with a term for all dementia to examine the mortality HR for total dementia.

Population attributable risk percentage: Excess deaths due to $A D$ dementia. We calculated population attributable risk percentage (PAR\%) to estimate the percentage of total mortality risk for the cohort that could be considered attributable to an incident diagnosis of $\mathrm{AD}$ dementia. PAR\% as calculated here is known as the "excess fraction" and is defined as the proportion of new cases of an outcome that occurs in the exposed group that is in excess of new cases in the unexposed group (for example, the proportion of lung cancer cases in smokers that is in excess of lung cancer cases in nonsmokers). ${ }^{22}$ In this study, the outcome is mortality and the exposure is incident $\mathrm{AD}$ dementia, so $\mathrm{PAR} \%$ represents the proportion of deaths that occur after developing $\mathrm{AD}$ that is in excess of deaths among people without AD. Crude PAR\% was calculated based on the mortality rate in the entire cohort $\left(\mathrm{I}_{t}\right)$ and mortality rate among the person-years without $\mathrm{AD}$ dementia over follow-up $\left(\mathrm{I}_{\mathrm{n}}\right)$ using the following formula:

$$
\operatorname{PAR} \%_{\text {crude }}=\left(\mathrm{I}_{\mathrm{t}}-\mathrm{I}_{\mathrm{n}}\right) / \mathrm{I}_{\mathrm{t}} \times 100 \%
$$

We then calculated an "adjusted PAR\%" using HRs from the adjusted proportional hazards models:

$$
\operatorname{PAR} \%_{\text {adjusted }}=\mathrm{p}(\mathrm{r}-1) /[(\mathrm{p} \times \mathrm{r})+(1-\mathrm{p})] \times 100 \%,
$$

where $\mathrm{p}$ is the prevalence (proportion with disease) of $\mathrm{AD}$ dementia and $r$ is the adjusted mortality $\mathrm{HR}$ for $\mathrm{AD}$ dementia. Because prevalence cannot be accurately calculated based on the design of the cohorts used for this study in which persons with known dementia were excluded, we used recent US prevalence estimates from a study that applied $\mathrm{AD}$ incidence estimates from the Chicago Health and Aging Project to 2010 US census data: $3.0 \%$ for ages $65-74$, $17.6 \%$ for ages $75-84$, and $32.3 \%$ for ages 85 and older. ${ }^{3}$ The Chicago Health and Aging Project, a population-based cohort study of older adults, used criteria for a diagnosis of $\mathrm{AD}$ dementia identical in all essential aspects to those used here.

Number of deaths attributable to AD dementia in the United States. We then applied our PAR\% estimates to the reported numbers of total US deaths in these age ranges for 2010. ${ }^{1}$ Because our estimates of HRs of mortality for incident $\mathrm{AD}$ dementia may not be representative of the US population, we performed a sensitivity analysis in which we calculated the number of US deaths attributable to AD dementia if the HRs for each age interval were lower or higher than the estimate from our cohort (by $10 \%$ intervals up to $50 \%$ lower or higher).

RESULTS Mortality rates and survival after AD dementia. Over an average of 8.0 years of follow-up per person and a total of 18,981 person-years, of 2,566 participants without dementia at baseline, $559(21.8 \%)$ persons 
Table 1 Characteristics of participants who did and did not develop AD

\begin{tabular}{|c|c|c|c|c|}
\hline & Total $(n=2,566)$ & No $A D(n=2,007)$ & Incident AD ( $n=559)$ & $p$ For difference ${ }^{a}$ \\
\hline Age at study baseline, $y$, mean (SD) & $78.07(7.37)$ & $77.27(7.36)$ & $80.94(6.64)$ & $<0.0001$ \\
\hline Years of follow-up, mean (SD) & $7.95(4.87)$ & $7.58(5.00)$ & $9.27(4.08)$ & $<0.0001$ \\
\hline Female sex, $n(\%)$ & $1,854(72.3)$ & $1,450(72.0)$ & $404(73.0)$ & 0.915 \\
\hline White, non-Hispanic, n (\%) & $2,272(88.7)$ & $1,762(88.0)$ & $510(92.0)$ & 0.015 \\
\hline Education, $y$, mean (SD) & $16.03(3.80)$ & $15.96(3.84)$ & $16.30(3.68)$ & 0.057 \\
\hline Died, n (\%) & $1,090(42.5)$ & 692 (34.5) & 398 (71.2) & $<0.0001$ \\
\hline
\end{tabular}

Abbreviation: $A D=$ Alzheimer disease.

Participants from the Memory and Aging Project and Religious Orders Study.

${ }^{a}$ Difference between no-AD and incident-AD groups; $p$ values from $\chi^{2}$ (categorical variables) or analysis of variance (continuous variables).

were diagnosed with $\mathrm{AD}$ dementia, $31(1.2 \%)$ were diagnosed with other forms of dementia, and 1,090 (42.4\%) died. The mean age at incident $\mathrm{AD}$ dementia diagnosis was $86.53(\mathrm{SD}=6.47)$ years. As shown in table 1, participants who developed $\mathrm{AD}$ dementia were older and were followed longer. Seventy-two percent of those who developed $\mathrm{AD}$ dementia died, whereas $34.5 \%$ of those who did not develop $\mathrm{AD}$ dementia died (table 1). The median survival time from $\mathrm{AD}$ dementia diagnosis to death from Kaplan-Meier curves for participants who developed $\mathrm{AD}$ dementia was 3.8 years overall and was related to age at diagnosis. Median survival was 4.4 years for persons aged $75-84$ at $\mathrm{AD}$ dementia diagnosis $(\mathrm{n}=$ 182) and 3.2 for persons aged 85 and older at $\mathrm{AD}$ dementia diagnosis $(\mathrm{n}=356)$; only 4 persons aged 65-74 died after developing AD dementia, so KaplanMeier curve could not be estimated.

\section{Figure Predicted survival past age 75 years after a diagnosis of AD dementia vs no diagnosis of $A D$ dementia}

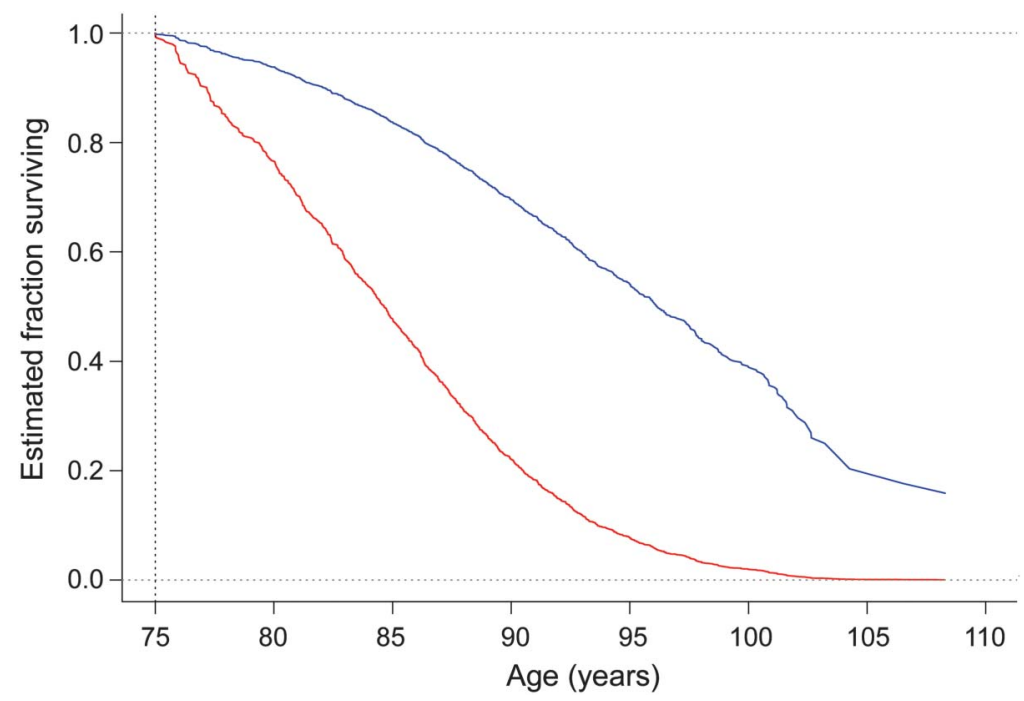

Estimates for white females with 15 years of education, Alzheimer disease (AD) diagnosed at age 75 years. Red = predicted survival after a diagnosis of $A D$; blue = predicted survival without $A D$.
$\mathrm{AD}$ dementia and risk of mortality. From a proportional hazards model adjusted for sex, race, education, and parent study with age as the time scale, the rate of mortality was more than 3 times higher after a diagnosis of $\mathrm{AD}$ dementia $(\mathrm{HR}=3.13, \mathrm{CI}=2.74$, 3.58; see the figure). After further adjustment for vascular disease and vascular risk burden, results were similar $(\mathrm{HR}=3.17, \mathrm{CI}=2.77,3.626)$; we therefore omitted vascular disease from subsequent analyses. Examining the data by age group, only 4 persons aged 65-74 died after developing AD dementia (table 2); therefore, HR estimates were unstable in this group. In persons aged 75-84, the rate of mortality was more than 4 times higher after a diagnosis of $\mathrm{AD}$ dementia, and in persons aged 85 and older, it was nearly 3 times higher after a diagnosis of $\mathrm{AD}$ dementia (table 2). We then repeated the models for any diagnosis of dementia. Results were similar for total dementia: $\mathrm{HR}=3.21$, $\mathrm{CI}=2.81,3.67$ across all ages; $\mathrm{HR}=4.54, \mathrm{CI}=$ $3.54,5.83$ for ages $75-84$; and $\mathrm{HR}=2.77, \mathrm{CI}=2.37$, 3.23 for ages 85 and older.

Population attributable risk percentage. Crude PAR\% was $16.9 \%$ for ages $75-84$ and $30.2 \%$ for ages 85 and older. Adjusted PAR\%, based on HRs from proportional hazards models and age-specific prevalence rates from the literature, ${ }^{3}$ was higher than crude PAR\% (table 2).

Number of deaths attributable to AD dementia in the United States. We applied age-specific estimates of PAR\% to the numbers of deaths in the United States in 10-year age groups ${ }^{1}$ and obtained a figure of 503,400 excess deaths after a diagnosis of $\mathrm{AD}$ dementia in 2010 for Americans aged 75 and older. We then performed a sensitivity analysis in which we calculated the number of excess deaths attributable to $\mathrm{AD}$ dementia in the United States if the mortality HR for $\mathrm{AD}$ dementia was lower or higher than that which was observed (table 3). For example, if the mortality HR for $\mathrm{AD}$ dementia was $20 \%$ lower than the observed HR in each age strata, the number of deaths attributable to 
Table 2 Attributable risk of $A D$ and on mortality estimates by age strata ${ }^{a}$
Ages 65-74 y Ages 75-84 y

AD prevalence (US estimate), ${ }^{\mathrm{b}} \%$

In age range at baseline

Years of follow-up (SD)

Developed AD, n (\%)

Deaths (deaths after $A D)^{c}$

Crude PAR\%

Adjusted PAR\% ${ }^{\circ}$



906

$8.58(5.41)$

115 (12.7)

57 (4)

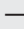

$-$
Mortality $\mathrm{HR}_{A D}{ }^{d}$
Ages 85 y and older

32.3

463

4.31 (3.38)

151 (32.6)

690 (311)

$343(83)$

$4.30(3.33,5.58)$

16.93

30.20

37.00

35.76
Abbreviations: $A D=$ Alzheimer disease; $\mathrm{HR}=$ hazard ratio; $\mathrm{PAR} \%$ = population attributable risk percentage.

${ }^{a}$ Because of the small number of persons who died after developing AD in the age 65-74 group (4), we do not present HRs or attributable risk estimates.

${ }^{\mathrm{b}}$ From literature for 2010 (Hebert et al., ${ }^{3}$ 2013).

${ }^{\mathrm{c}}$ Refers to deaths occurring in that age range (participant may have started study in earlier age range); deaths in that age range after the occurrence of $A D$ are presented in parentheses.

${ }^{\mathrm{d}}$ Hazard of mortality after $A D$ compared with no $A D$ from models adjusted for sex, race, education, and parent study. Data presented as estimate (confidence interval).

${ }^{\mathrm{e}}$ Based on adjusted HR and AD prevalence.

$\mathrm{AD}$ dementia in the United States in those aged 75 and older in 2010 would be approximately 400,000. By contrast, if the HR was $20 \%$ higher, the number of deaths would be nearly 600,000 .

DISCUSSION In this study of older adults without dementia at the beginning of observation, the development of $\mathrm{AD}$ dementia significantly contributed to the burden of mortality. The rate of death was 3 to 4 times higher after a diagnosis of $\mathrm{AD}$ dementia and more than one-third of all deaths were attributable to $\mathrm{AD}$ dementia. This translates into a figure of more than 500,000 deaths attributable to AD dementia in the US population in 2010. Overall, the data indicate that the proportion of older persons who die of $\mathrm{AD}$ is much higher than the number indicated by death certificates, which is less than $5 \%$ of all deaths in the elderly. ${ }^{1}$

Some of our results are comparable to other cohort studies. Our median survival time after incident AD dementia was similar to other reports, ranging from 3.1 to 5.9 years. ${ }^{2,9,12,13,23}$ Our cohorts were older than other cohorts; studies presenting median survival time by age categories had similar findings. ${ }^{5,24,25}$ Our observed mortality $\mathrm{HR}$ for $\mathrm{AD}$ dementia was similar to other studies, ranging from 1.7 to 3.7. ${ }^{7,8,13}$ Our estimates of PAR\%, however, were higher than earlier estimates, which ranged from $12.5 \%$ to $18.3 \% .{ }^{29,10}$ A possible explanation is that previous studies without annual assessments may have underestimated mortality associated with $\mathrm{AD}$ dementia because of "length bias" from long intervals between assessments; i.e., persons with rapidly progressive $\mathrm{AD}$ die before being diagnosed. ${ }^{12,13}$ Our cohort may also be healthier and thus $\mathrm{AD}$ may be involved in proportionately more deaths given fewer comorbidities. Finally, a 1995 study using a lower HR for mortality reported 163,000 to 173,000 deaths attributable to $\mathrm{AD}$ dementia in the United States. ${ }^{26}$

The cohorts used in this analysis may differ in systematic ways from the general US population of older adults, in particular because of better health and more education. This would influence the up-weighting of our estimates to national mortality figures in order to estimate the number of deaths attributable to $\mathrm{AD}$ dementia in the United States. However, our estimates provide an approximate sense of the magnitude of the underestimation of deaths attributable to $\mathrm{AD}$ if relying on death certificates alone. Although our estimate of 503,400 deaths attributable to $\mathrm{AD}$ is crude, it is notably 5 to 6 times higher than the 83,494 AD deaths in 2010 reported by the CDC. ${ }^{1}$ Our figure suggests that $\mathrm{AD}$ may be the third leading cause of death after heart disease and cancer, with nearly as many deaths as chronic lower respiratory diseases, stroke, and accidents (causes 3-5 as listed by the CDC) combined, ${ }^{1}$ echoing a recent report indicating that dementia has an impact on survival in the range of cardiovascular disease. ${ }^{23}$ Sensitivity analyses showed that even if our estimates of hazard of death were off by a factor of 2 , this figure would be approximately 200,000 , which is still substantially higher than the figure from the CDC.

It is well documented that $\mathrm{AD}$ and other forms of dementia are underreported on death certificates. ${ }^{27-30}$ Dementia contributes to death insidiously over the course of years through a cascade of events. Eventually, severe dementia causes complications such as swallowing disorders and malnutrition that can lead to fatal

Table 3 Estimated number of deaths attributable to Alzheimer disease in the United States in $2010^{\circ}$

\begin{tabular}{|c|c|c|c|c|c|c|c|c|c|c|c|}
\hline \multirow[b]{2}{*}{ Age group, y } & \multicolumn{11}{|c|}{ Percentage of age-specific hazard ratio used for calculation } \\
\hline & $50 \%$ & $60 \%$ & $70 \%$ & $80 \%$ & $90 \%$ & $100 \%$ & $110 \%$ & $120 \%$ & $130 \%$ & $140 \%$ & $150 \%$ \\
\hline 75-84 & 105,300 & 136,100 & 163,500 & 188,000 & 210,000 & 229,900 & 248,000 & 264,500 & 279,600 & 293,500 & 306,300 \\
\hline $85+$ & 78,600 & 129,000 & 172,600 & 210,500 & 243,900 & 273,500 & 299,900 & 323,600 & 345,100 & 364,500 & 382,200 \\
\hline Subtotal (age 75+) & 183,900 & 265,200 & 336,100 & 398,500 & 453,900 & 503,400 & 547,900 & 588,000 & 624,600 & 658,000 & 688,500 \\
\hline
\end{tabular}

Sensitivity analysis based on percentage of mortality hazard ratios for incident Alzheimer disease.

${ }^{a}$ Each figure rounded to nearest 100. 
conditions such as pneumonia. ${ }^{31,32}$ These more proximate causes are listed on the death certificate as immediate cause of death, while dementia is often omitted as an underlying cause. Attempting to identify a single cause of death may not capture the reality of the process of dying for most elderly people because multiple factors may contribute to death in the elderly, some proximate and some distal. The elimination of any one of them may allow the individual to live longer. Just as the field has embraced the concept of mixed dementia, acknowledging that multiple neuropathologies may contribute to the expression of dementia beyond $\mathrm{AD}$ pathology alone, ${ }^{33}$ it may be time to consider the analogous concept of "mixed mortality" to more accurately reflect the contribution of multiple disease processes to dying. This more nuanced view of "cause of death" is needed for an accurate understanding of the contributions of chronic diseases such as $\mathrm{AD}$ to death in rapidly aging populations.

There are several limitations to this work. Because these cohort studies are not population-based and participants agree to autopsy, mortality rates and attributable risk may not be representative of the general population. Our results may be biased if the relationship between $\mathrm{AD}$ dementia and death is different than for the general US population of older adults. Although we adjusted for potential confounders of the relationship between $\mathrm{AD}$ dementia and death, residual confounding may remain. We were only able to estimate deaths attributable to $\mathrm{AD}$ dementia for persons aged 75 years and older, and we did not include deaths associated with mild cognitive impairment due to $\mathrm{AD}$ as recognized by the newly recommended diagnostic criteria for clinical $\mathrm{AD},{ }^{34}$ so we likely underestimated the true number of deaths attributable to $\mathrm{AD}$; prior work has found that mild cognitive impairment is associated with mortality. ${ }^{18,35}$ Another limitation was the use of prevalence estimates from a separate cohort, calculated using different incidence rates, death rates, and lower HRs for mortality. ${ }^{3}$ Sensitivity analyses included halving our HR to lower than that assumed by the prevalence calculations in that study, so they are more conservative than required to overcome this limitation. $\mathrm{AD}$ dementia status was treated as an absorbing state, but it is possible for persons to not receive a diagnosis on subsequent assessments. Because most will eventually transition back into $\mathrm{AD}$ dementia, we chose not to complicate analyses by allowing such transitions. Finally, PAR\% calculations have been criticized because the sum of PAR\% estimates for different factors on the same outcome is often larger than $100 \%$. However, this conforms to our concept of "mixed mortality"; indeed, many of the deaths attributable to $\mathrm{AD}$ dementia may also be attributable to other co-occurring conditions.

This study has a number of strengths. There are few large studies of this kind with annual clinical evaluations, robust standardized diagnostic criteria, and very high rates of follow-up of living participants. Based on this study design, these results have a high level of internal validity and results are not likely to be biased because of selective attrition. Developing a consensus around the burden of mortality imposed by $\mathrm{AD}$ and dementia is important for informing government and private research priorities. The estimates generated by this analysis suggest that deaths from $\mathrm{AD}$ far exceed the numbers reported by the CDC and may be closer in magnitude to the number of deaths reported for heart disease and cancer.

\section{AUTHOR CONTRIBUTIONS}

Dr. Bryan D. James is the primary author of this manuscript. He was responsible for the study concept and design, interpretation of data, and drafting the manuscript. Dr. Sue E. Leurgans conducted the analysis for this study and contributed to the study concept and design and interpretation of data. Dr. Liesi E. Hebert, Dr. Paul A. Scherr, and Dr. Kristine Yaffe contributed to the study design and interpretation of data. Dr. David A. Bennett is the senior author of this manuscript. He is responsible for the initial concept of the study, study supervision, contribution to interpretation of data, and obtaining funding for the cohort studies.

\section{ACKNOWLEDGMENT}

The authors thank the nuns, priests, and brothers from across the country participating in the Religious Orders Study, the individuals from across northeastern Illinois participating in the Rush Memory and Aging Project, and the staff of the Rush Alzheimer's Disease Center. This work was motivated in part by a series of AD Epidemiology Meetings held in 2011-2012. The meetings were chaired by Dr. Stanley Prusiner with support from the Vradenburg Foundation. Meeting participants included Drs. David Bennett, Denis Evans, Bryan James, Kenneth Langa, Stanley Prusiner, Paul Scherr, David Weir, and Kristine Yaffe, and Mr. George Vradenburg.

\section{STUDY FUNDING}

Directly supported by the National Institute on Aging (P30 AG10161, R01AG15819, R01AG17917, K24AG031155), and the Illinois Department of Public Health (contract 23282007).

\section{DISCLOSURE}

B. James is funded by National Institute on Aging (NIA) grants R01AG17917, R01AG033678, and P30 AG10161. Dr. James is a consultant for the Alzheimer's Association and Partner's Health Care System. S. Leurgans is supported by grants from the NIH (R01AG017917, P30AG010161, RC2AG036547, R01AG042210, P20MD006886, R01NS078009, R01AG036042, P01AG014449, P01AG009466, R01NS028127, and R21AG030346) and the Illinois Department of Public Health (contract 23282007). Dr. Leurgans served as a paid faculty member for a Parkinson's Disease Foundation Learning Institute. L. Hebert is/has been supported by NIH grants NR010211, AG303544, AG011101, AG036650, and AG009966. Dr. Hebert reports no disclosures. P. Scherr is supported as a consultant to the Rush Institute of Healthy Aging. Dr. Scherr reports no disclosures. K. Yaffe is supported by NIA K24 AG031155. Dr. Yaffe reports research support from the NIH (NIA R01 AG05401, NIMH R01 MH086498, NIA R01 AG026720, NIDDK R01 DK069406, NIA P50 AG023501, and NIA 2P01 AG019724), the Alzheimer's Association, Department of Defense, California Department of Public Health, and the Bright Focus Foundation. She serves on data safety monitoring boards for Takeda, Inc. and a study sponsored by the NIH and has served as a consultant for Novartis, Inc. D. Bennett is supported by grants from the NIH (R01AG017917, P30AG010161, R01AG015819, U01AG46152, and R01AG036042), and Nutricia, Inc. Dr. Bennett serves on the editorial board of Neurology, Current Alzheimer Research, and Neuroepidemiology; has received honoraria for nonindustry-sponsored lectures; serves on data safety monitoring boards for Takeda, Inc., and has served as a consultant to Nutricia, Inc., Eli Lilly, Inc., Gerson Lehrman Group, Double Helix, and Enzymotic, Ltd. Go to Neurology.org for full disclosures.

Received June 12, 2013. Accepted in final form December 11, 2013. 


\section{REFERENCES}

1. Murphy SL, Xu J, Kochanek KD. Deaths: final data for 2010. In: National Vital Statistics Reports [online]. Available at: http://www.cdc.gov/nchs/data/nvsr/nvsr61/nvsr61_04.pdf. Accessed November 27, 2013.

2. Ganguli M, Dodge HH, Shen C, Pandav RS, DeKosky ST. Alzheimer disease and mortality: a 15-year epidemiological study. Arch Neurol 2005;62:779-784.

3. Hebert LE, Weuve J, Scherr PA, Evans DA. Alzheimer disease in the United States (2010-2050) estimated using the 2010 census. Neurology 2013;80:1778-1783.

4. Plassman BL, Langa KM, Fisher GG, et al. Prevalence of dementia in the United States: the aging, demographics, and memory study. Neuroepidemiology 2007;29:125-132.

5. Brookmeyer R, Corrada MM, Curriero FC, Kawas C. Survival following a diagnosis of Alzheimer disease. Arch Neurol 2002;59:1764-1767.

6. U.S. Department of Health and Human Services. National plan to address Alzheimer's disease: 2013 update. Available at: http://aspe.hhs.gov/daltcp/napa/NatlPlan2013.pdf. Accessed November 27, 2013.

7. Rait G, Walters K, Bottomley C, Petersen I, Iliffe S, Nazareth I. Survival of people with clinical diagnosis of dementia in primary care: cohort study. BMJ 2010;341:c3584.

8. Wilson RS, Aggarwal NT, Barnes LL, Bienias JL, Mendes de Leon CF, Evans DA. Biracial population study of mortality in mild cognitive impairment and Alzheimer disease. Arch Neurol 2009;66:767-772.

9. Aguero-Torres $\mathrm{H}$, Fratiglioni L, Guo Z, Viitanen M, Winblad B. Mortality from dementia in advanced age: a 5 -year follow-up study of incident dementia cases. J Clin Epidemiol 1999;52:737-743.

10. Tschanz JT, Corcoran C, Skoog I, et al. Dementia: the leading predictor of death in a defined elderly population: the Cache County Study. Neurology 2004;62:1156-1162.

11. Valcour VG, Masaki KH, Curb JD, Blanchette PL. The detection of dementia in the primary care setting. Arch Intern Med 2000;160:2964-2968.

12. Wolfson C, Wolfson DB, Asgharian M, et al. A reevaluation of the duration of survival after the onset of dementia. N Engl J Med 2001;344:1111-1116.

13. Helmer C, Joly P, Letenneur L, Commenges D, Dartigues JF. Mortality with dementia: results from a French prospective community-based cohort. Am J Epidemiol 2001;154:642-648.

14. Bennett DA, Schneider JA, Arvanitakis Z, Wilson RS. Overview and findings from the Religious Orders Study. Curr Alzheimer Res 2012;9:628-645.

15. Bennett DA, Schneider JA, Buchman AS, Barnes LL, Boyle PA, Wilson RS. Overview and findings from the Rush Memory and Aging Project. Curr Alzheimer Res 2012;9:646-663.

16. Bennett DA, Schneider JA, Aggarwal NT, et al. Decision rules guiding the clinical diagnosis of Alzheimer's disease in two community-based cohort studies compared to standard practice in a clinic-based cohort study. Neuroepidemiology 2006;27:169-176.

17. Bennett DA, Schneider JA, Buchman AS, Mendes de Leon C, Bienias JL, Wilson RS. The Rush Memory and Aging Project: study design and baseline characteristics of the study cohort. Neuroepidemiology 2005;25:163-175.
18. Bennett DA, Wilson RS, Schneider JA, et al. Natural history of mild cognitive impairment in older persons. Neurology 2002;59:198-205.

19. McKhann G, Drachman D, Folstein M, Katzman R, Price D, Stadlan EM. Clinical diagnosis of Alzheimer's disease: report of the NINCDS-ADRDA Work Group under the auspices of Department of Health and Human Services Task Force on Alzheimer's Disease. Neurology 1984;34:939-944.

20. Schneider JA, Arvanitakis Z, Leurgans SE, Bennett DA. The neuropathology of probable Alzheimer disease and mild cognitive impairment. Ann Neurol 2009;66:200-208.

21. Therneau TM, Grambsch PM. Modeling Survival Data: Extending the Cox Model, 1st ed. New York: SpringerVerlag; 2010.

22. Greenland S, Robins JM. Conceptual problems in the definition and interpretation of attributable fractions. Am J Epidemiol 1988;128:1185-1197.

23. Rizzuto D, Bellocco R, Kivipelto M, Clerici F, Wimo A, Fratiglioni L. Dementia after age 75: survival in different severity stages and years of life lost. Curr Alzheimer Res 2012;9: 795-800.

24. Helzner EP, Scarmeas N, Cosentino S, Tang MX, Schupf N, Stern Y. Survival in Alzheimer disease: a multiethnic, population-based study of incident cases. Neurology 2008; 71:1489-1495.

25. Larson EB, Shadlen MF, Wang L, et al. Survival after initial diagnosis of Alzheimer disease. Ann Intern Med 2004;140:501-509.

26. Ewbank DC. Deaths attributable to Alzheimer's disease in the United States. Am J Public Health 1999;89:90-92.

27. Ives DG, Samuel P, Psaty BM, Kuller LH. Agreement between nosologist and cardiovascular health study review of deaths: implications of coding differences. J Am Geriatr Soc 2009;57:133-139.

28. Ganguli M, Rodriguez EG. Reporting of dementia on death certificates: a community study. J Am Geriatr Soc 1999;47:842-849.

29. Olichney JM, Hofstetter CR, Galasko D, Thal LJ, Katzman R. Death certificate reporting of dementia and mortality in an Alzheimer's disease research center cohort. J Am Geriatr Soc 1995;43:890-893.

30. Macera CA, Sun RK, Yeager KK, Brandes DA. Sensitivity and specificity of death certificate diagnoses for dementing illnesses, 1988-1990. J Am Geriatr Soc 1992;40:479-481.

31. Burns A, Jacoby R, Luthert P, Levy R. Cause of death in Alzheimer's disease. Age Ageing 1990;19:341-344.

32. Brunnstrom HR, Englund EM. Cause of death in patients with dementia disorders. Eur J Neurol 2009;16:488-492.

33. Schneider JA, Arvanitakis Z, Bang W, Bennett DA. Mixed brain pathologies account for most dementia cases in community-dwelling older persons. Neurology 2007;69: 2197-2204.

34. Albert MS, DeKosky ST, Dickson D, et al. The diagnosis of mild cognitive impairment due to Alzheimer's disease: recommendations from the National Institute on Aging-Alzheimer's Association workgroups on diagnostic guidelines for Alzheimer's disease. Alzheimers Dement 2011;7:270-279.

35. Bassuk SS, Wypij D, Berkman LF. Cognitive impairment and mortality in the community-dwelling elderly. Am J Epidemiol 2000;151:676-688. 